International Mathematical Forum, 2, 2007, no. 3, 137 - 142

\title{
On the instability of positive solution of an elliptic equation
}

\author{
G. A. Afrouzi and S. H. Rasouli \\ Department of Mathematics, Faculty of Basic Sciences \\ Mazandaran University, Babolsar, Iran \\ afrouzi@umz.ac.ir
}

\begin{abstract}
We study the stability of positive stationary solutions of

$$
\begin{cases}-\Delta u(x)=\lambda f(x, u), & x \in \Omega \\ B u=0, & x \in \partial \Omega\end{cases}
$$

where $\Omega$ is a bounded domain in $R^{n}$ with smooth boundary $B u(x)=$ $\alpha h(x) u+(1-\alpha) \frac{\partial u}{\partial n}$ where $\alpha \in[0,1], h: \partial \Omega \longrightarrow R^{+}$with $h=1$ when $\alpha=1, \lambda>0, f$ is a smooth function such that $f_{u u}(x, u)>0$ for all fixed $x \in \Omega\left(u \in R^{+}\right), f_{x}(0,0)=0, f(x, u)<0$ for $u \in(0, \beta)$ and $f(x, u)>0$ for $u>\beta$ for some $\beta>0$ (for all fixed $x \in \Omega$ ). We provide a simple proof to establish that every positive stationary solution is linearly unstable.
\end{abstract}

Keywords: Instability, linearized equation, positive solutions

Mathematics Subject Classification: 35B35, 35J65

\section{Introduction}

In this paper, we consider the stability of positive stationary solutions to the elliptic boundary value problem

$$
\begin{array}{lr}
-\Delta u(x)=\lambda f(x, u), & x \in \Omega, \\
B u=0, & x \in \partial \Omega,
\end{array}
$$

where $\Omega$ is a bounded domain in $R^{n}$ with smooth boundary $B u(x)=\alpha h(x) u+$ $(1-\alpha) \frac{\partial u}{\partial n}$ where $\alpha \in[0,1], h: \partial \Omega \longrightarrow R^{+}$is a smooth function with $h=1$ when $\alpha=1$, i.e., the boundary condition may be of Dirichlet, Neumann or mixed type, $\lambda>0$ is a constant and $f$ is a smooth function satisfying 


$$
f_{u u}(x, u)>0 \text { for all fixed } x \in \Omega\left(u \in R^{+}\right) \text {and } f_{x}(0,0)=0,
$$

and

$$
f(x, u)<0 \text { for } u \in(0, \beta) \text { and } f(x, u)>0 \text { for } u>\beta \text { for some } \beta>0 \text {, }
$$

for all fixed $x \in \Omega$, where $f_{u}(x, u)$ denotes the partial derivative of $f(x, u)$ with respect to $u$.

In the case when $f(x, u) \equiv f(u)$, was studied by several authors. Brown and his co-authors have altogether proved that if $f^{\prime \prime}>0$ and $f(0) \leq 0$, then every non-trivial nonnegative solution of the problem

$$
\begin{cases}-\Delta u=\lambda f(u), & x \in \Omega, \\ u=0, & x \in \partial \Omega,\end{cases}
$$

is unstable. They first considered the monotone case, i.e., $f^{\prime}>0$ in [2]. The non-monotone case was first proved by Tertikas [4] using sub- and supersolution. For the case $f(x, u) \equiv m(x) u(u-1)$, Afrouzi and Rasouli [1] studied the instability of positive solutions. The purpose of this paper is to extend this study to problem (1). The main result of [2] is summarized in the following theorem.

Theorem 1.1. Let $f: R \longrightarrow R$ be a twice continuously differentiable function, then

(i) if $f^{\prime \prime}>0$ and $f(0) \leq 0$, then every nontrivial nonnegative solution of (5) is unstable. while

(ii) if $f^{\prime \prime}<0$ and $f(0) \geq 0$, then every nontrivial nonnegative solution of (5) is stable.

We recall that, if $u$ be any nonnegative solution of

$$
\begin{cases}-\Delta u=g(x, u), & x \in \Omega \\ u=0, & x \in \partial \Omega\end{cases}
$$

then the linearized equation about $u$ is

$$
\begin{cases}-\Delta \phi-g_{u}(x, u) \phi=\mu \phi, & x \in \Omega \\ \phi=0, & x \in \partial \Omega .\end{cases}
$$

Definition 1.3. We call a solution $u$ of (6) a linearly stable solution if all eigenvalues of (7) are strictly positive, which can be inferred if the principal eigenvalue $\mu_{1}>0$. Otherwise $u$ is linearly unstable. 


\section{Main result}

In this section we shall prove the instability of positive stationary solution $u$ by showing that the principal eigenvalue $\mu_{1}$, of the equation linearized about $u$ is negative; the stability of $u$ then follows from the well-known principle of linearized stability (see [3]). We overcome the difficulty of $f$ being nonmonotone, by re-writing $f$ as the sum of the monotone function and a linear function involving $f(0,0)$ and $f_{u}(0,0)$. By doing so we arrive at a much simpler proof clearly indicating the role of $f(0,0)$ in establishing the instability result. Our main result is the following theorem.

Theorem 1.1 : Every positive stationary solution of (1)-(2) is linearly unstable.

Proof. Let $g(x, u)=f(x, u)-f(0,0)+\left|f_{u}(0,0)\right| u$. Then $g(x, 0)=0$ for all fixed $x \in \Omega, g_{u}(x, u)=f_{u}(x, u)+\left|f_{u}(0,0)\right|, g_{u u}(x, u)=f_{u u}(x, u)>0$ for all fixed $x \in \Omega\left(u \in R^{+}\right)$and, therefore $\left.g_{u}(x, u)\right)>0$ for all fixed $x \in \Omega\left(u \in R^{+}\right)$ and $g(x, u)>0$ for all fixed $x \in \Omega\left(u \in R^{+}\right)$. Now, (1) $-(2)$ can be rewritten as

$$
\begin{gathered}
-\Delta u(x)=\lambda\left\{g(x, u)+f(0,0)-\left|f_{u}(0,0)\right| u\right\}, \quad x \in \Omega, \\
B u(x)=0, \quad x \in \partial \Omega .
\end{gathered}
$$

Let $u_{0}$ be any positive stationary solution of $(8)-(9)$. Then the linearized equation about $u_{0}$ is

$$
\begin{gathered}
-\Delta \phi(x)-\lambda\left\{g_{u}\left(x, u_{0}\right)-\left|f_{u}(0,0)\right|\right\} \phi(x)=\mu \phi(x), \quad x \in \Omega, \\
B \phi(x)=0, \quad x \in \partial \Omega,
\end{gathered}
$$

Let $\mu_{1}$ be the principal eigenvalue and let $\psi(x)(\geq 0)$ be a corresponding eigenfunction. Multiplying (8) by $g_{u}\left(x, u_{0}\right) \psi(x)$ and $(10)$ by $g\left(x, u_{0}\right)$, then subtracting and integrating over $\Omega$, we obtain

$$
\begin{gathered}
\int_{\Omega}\left\{\left(-\Delta u_{0}\right) g_{u}\left(x, u_{0}\right) \psi(x)-(-\Delta \psi(x)) g\left(x, u_{0}\right)-\lambda f(0,0) g_{u}\left(x, u_{0}\right) \psi(x)\right. \\
\left.+\lambda\left|f_{u}(0,0)\right| u_{0} \psi(x)-\lambda\left|f_{u}(0,0)\right| g\left(x, u_{0}\right) \psi(x)\right\} d x \\
=-\mu_{1} \int_{\Omega} \psi(x) g\left(x, u_{0}\right) d x
\end{gathered}
$$


But by Green's first identity, we have

$$
\begin{gathered}
\int_{\Omega}\left(-\Delta u_{0}\right) g_{u}\left(x, u_{0}\right) \psi(x) d x=\int_{\Omega} \nabla\left(g_{u}\left(x, u_{0}\right) \psi(x)\right) \nabla u_{0}(x) d x \\
-\int_{\partial \Omega} g_{u}\left(x, u_{0}\right) \psi(s)\left(\frac{\partial u_{0}}{\partial n}\right) d s=\int_{\Omega} g_{u u}\left(x, u_{0}\right) \psi(x)\left|\nabla u_{0}\right|^{2} d x \\
\quad+\int_{\Omega} g_{u}\left(x, u_{0}\right)\left(\nabla \psi \nabla u_{0}\right) d x-\int_{\partial \Omega} g_{u}\left(x, u_{0}\right) \psi(s)\left(\frac{\partial u_{0}}{\partial n}\right) d s
\end{gathered}
$$

and

$$
\begin{gathered}
\int_{\Omega}(\Delta \psi(x)) g\left(x, u_{0}\right) d x=-\int_{\Omega} \nabla\left(g\left(x, u_{0}\right)\right) \nabla \psi(x) d x+\int_{\partial \Omega} g\left(x, u_{0}\right)\left(\frac{\partial \psi}{\partial n}\right) d s \\
=-\int_{\Omega} g_{u}\left(x, u_{0}\right)\left(\nabla u_{0} \nabla \psi\right) d x+\int_{\partial \Omega} g\left(x, u_{0}\right)\left(\frac{\partial \psi}{\partial n}\right) d s .
\end{gathered}
$$

By using (13) - (14) in (12) we get

$$
\begin{gathered}
-\mu_{1} \int_{\Omega} \psi(x) g\left(x, u_{0}\right) d x=\int_{\Omega} g_{u u}\left(x, u_{0}\right) \psi(x)\left|\nabla u_{0}\right|^{2} d x \\
-\lambda f(0,0) \int_{\Omega} g_{u}\left(x, u_{0}\right) \psi(x) d x+\lambda\left|f_{u}(0,0)\right| \int_{\Omega}\left\{g_{u}\left(x, u_{0}\right) u_{0}-g\left(x, u_{0}\right)\right\} \psi(x) d x \\
+\int_{\partial \Omega}\left\{g\left(x, u_{0}\right)\left(\frac{\partial \psi}{\partial n}\right)-g_{u}\left(x, u_{0}\right) \psi(s)\left(\frac{\partial \psi}{\partial n}\right)\right\} d s .
\end{gathered}
$$

We notice that when $\alpha=1$ (then $h=1$ ) we have $u_{0}=0$ for $s \in \partial \Omega$ and, therefore, $g\left(x, u_{0}\right)=0$ for $s \in \partial \Omega$ and also we have $\psi=0$ for $s \in \partial \Omega$. Hence,

$$
\int_{\partial \Omega}\left\{g\left(x, u_{0}\right)\left(\frac{\partial \psi}{\partial n}\right)-g_{u}\left(x, u_{0}\right) \psi(s)\left(\frac{\partial \psi}{\partial n}\right)\right\} d s=0,
$$

and when $\alpha \neq 1$, we have

$$
\begin{gathered}
\int_{\partial \Omega}\left\{g\left(x, u_{0}\right)\left(\frac{\partial \psi}{\partial n}\right)-g_{u}\left(x, u_{0}\right) \psi(s)\left(\frac{\partial \psi}{\partial n}\right)\right\} d s \\
=\int_{\partial \Omega}\left\{g\left(x, u_{0}\right)\left(\frac{(-\alpha h(x) \psi(x))}{(1-\alpha)}\right)-g_{u}\left(x, u_{0}\right) \psi(s)\left(\frac{\left(-\alpha h(x) u_{0}\right)}{(1-\alpha)}\right)\right\} d s
\end{gathered}
$$




$$
=\int_{\partial \Omega}\left\{\frac{\alpha h(x) \psi(s))}{(1-\alpha)}\right\}\left[u_{0} g_{u}\left(x, u_{0}\right)-g\left(x, u_{0}\right)\right] d s .
$$

But $\alpha \geq 0, h>0, \psi \geq 0$ for $s \in \partial \Omega$ and $u_{0} g_{u}\left(x, u_{0}\right)-g\left(x, u_{0}\right)>0$ for all fixed $x \in \Omega\left(u_{0} \in R^{+}\right)$. Therefore, if $\alpha \neq 1$

$$
\int_{\partial \Omega}\left\{\frac{\alpha h(x) \psi(s))}{(1-\alpha)}\right\}\left[u_{0} g_{u}\left(x, u_{0}\right)-g\left(x, u_{0}\right)\right] d s \geq 0 .
$$

Also, since $g_{u u}\left(x, u_{0}\right)>0$ for all fixed $x \in \Omega\left(u_{0} \in R^{+}\right)$, we get

$$
\int_{\Omega} g_{u u}\left(x, u_{0}\right) \psi(x)\left|\nabla u_{0}\right|^{2} d x>0
$$

Thus, by $(16)-(18)$ we have

$$
\begin{gathered}
-\mu_{1} \int_{\Omega} \psi(x) g\left(x, u_{0}\right) d x>-\lambda f(0,0) \int_{\Omega} g_{u}\left(x, u_{0}\right) \psi(x) d x \\
\quad+\lambda\left|f_{u}(0,0)\right| \int_{\Omega}\left\{g_{u}\left(x, u_{0}\right) u_{0}-g\left(x, u_{0}\right)\right\} \psi(x) d x .
\end{gathered}
$$

Now by using $(3)-(4)$ and the fact that $g_{u}\left(x, u_{0}\right) u_{0}-g\left(x, u_{0}\right)>0$ for all fixed $x \in \Omega$

$\left(u_{0} \in R^{+}\right)$in $(19)$ it is easy to see that

$$
-\mu_{1} \int_{\Omega} \psi(x) g\left(x, u_{0}\right) d x>0 .
$$

But $\psi>0$ for $x \in \Omega$ and $g\left(x, u_{0}\right)>0$ for all fixed $x \in \Omega\left(u_{0} \in R^{+}\right)$and hence, $\mu_{1}<0$ and the result follows (see [3]).

\section{References}

[1] G.A. Afrouzi, S.H. Rasouli. Instability of nonnegative solutions for a boundary value problem with indefinite weight function. Global. J. Pure. Appl. Math. Vol 1, no. 1, (2005), pp. 9-12.

[2] K.J. Brown, R. Shvaji. Instability of nonnegative solution for a class of semipositone problems. Proc. Amer. Math. Soc., 112 (1) (1991), pp. 121124. 
[3] D.H. Sattinger. Monotone methods in nonlinear elliptic and parabolic boundary value problems, Indiana Univ. Math. J., 21 (11)(1972), pp. 979-1000.

[4] A. Tertikas. Stability and instability of positive soulutions of semi-positone problems, Proc. AMS 114(4)(1992), pp. 1035-1040.

Received: March 1, 2006 